\title{
Identification of a Generator Fault by Model-Based Diagnostic Techniques
}

\author{
P. Pennacchi and A. Vania \\ Dipartimento di Meccanica, Politecnico di Milano, Milano, Italy
}

Model-based diagnostic techniques can be used successfully in the health analysis of rotormachinery. Unfortunately, a poor accuracy of the model of the fully assembled machine, as well as noise in the signals and errors in the evaluation of the experimental vibrations that are caused only by the impending fault, can affect the accuracy of the fault identifications. This can make it difficult to identify the type of actual fault as well as to evaluate with care its severity and position. This article shows some techniques that have been developed by the authors to measure the accuracy of the results obtained with model-based identification methods aimed to diagnose faults in rotating machines. In this article, the results obtained by means of the analysis of experimental data collected in a power plant are described. Finally, the capabilities of the developed methods are shown and discussed.

Keywords Accuracy indexes, Diagnostics, Identification, Rotor bow, Rotordynamics

Permanent condition monitoring can assure the early detection of faults and malfunctions in rotating machines. This can be very useful to prevent serious damage as well as to reduce the repair costs. However, in order to plan suitable maintenance actions, a fault diagnosis is required. A preliminary evaluation of the most probable types of fault can be obtained with a fault symptom analysis. However, more significant information can be provided by model-based diagnostic techniques (Markert et al., 2000; Platz et al., 2000; Bachschmid et al., 1999; Bachschmid and Pennacchi, 2000; Bachschmid et al., 2000). These methods allow the faults to be identified and localized as well as the fault severity to be evaluated.

Received 25 June 2002; accepted 1 July 2002.

Address correspondence to P. Pennacchi, Dipartimento di Meccanica, Politecnico di Milano, Campus Bovisa Via la Masa, 34, Milano, I-20158, Italy. E-mail: paolo.pennacchi@polimi.it
Usually, the model of the fully assembled machine allows the response of the rotors, bearings, and the foundation structure to be simulated. The rotor train can be modeled with beam finite elements (FE), while the dynamic effects of the faults can be simulated with suitable sets of equivalent forces and moments that are applied to the nodes of the FE model of the rotors. Therefore, the identification of a fault can be obtained by evaluating the system of excitations that minimizes the error between the machine experimental response and the numerical response evaluated with the model. Usually, this error is called residue. In order to make the comparison among the results of different analyses easier, a relative residue is evaluated.

Weighted least squares methods can be used to identify the equivalent forces and moments that simulate the faults. Although the vibrations of the shafts along the spans between two adjacent supports can give very useful information to identify the machine faults, usually, in real machines, only shaft and support vibrations measured on the journal bearings are available. This requires more careful fault identification analyses to be carried out.

Unfortunately, also in the absence of faults, the rotating machines are subjected to vibrations that, generally, are caused by a residual unbalance of the rotors as well as by misalignments of couplings and supports. These excitations cannot be easily modeled as they are unknown. Therefore, fault identification techniques require one to evaluate the transient vibrations of the machine obtained by subtracting the vibrations measured in normal state from those measured after the fault occurrence. If the non linearity of the system is negligible, these transient additional vibrations represent the machine response caused only by the fault. However, if the two speed transients occur in different thermal conditions of the machine or if other factors, in addition to the malfunction, affect the system vibrations, the accuracy of the results of the fault identification can be poor. Similar effects can be caused by the presence of noise in the vibration signals. Moreover, the accuracy of the results of modelbased techniques can be significantly influenced by the care with which the model of the fully assembled machine has been developed. Usually, a preliminary careful tuning of the model is 
required before applying it in detailed investigations. Owing to a poor accuracy of the experimental response, as well as of the model, the residues associated with different identified faults can be fairly similar. This can make it difficult to diagnose the type of actual fault as well as to evaluate its severity and position.

Therefore, some methods have been developed by the authors to measure the accuracy of the results obtained with modelbased diagnostic techniques aimed to identify faults in rotating machines. The information provided by these methods can be very useful to evaluate which of the identified faults has the highest probability of being the actual one. A first validation of these methods has been carried out using only ideal and corrupted machine responses simulated with mathematical models. The results of these investigations have been shown in previous publications (Pennacchi and Vania, 2001a; Pennacchi and Vania, 2001b). Conversly, this article shows the results obtained by the identification of a machine malfunction that has been detected on the generator of a co-generation unit. A non ideal tuning of the model of the fully assembled machine, as well as the necessity of subtracting the effects of the residual unbalance of the rotor from the transient responses, have affected in part the accuracy of the fault identification. However, the techniques developed by the authors to analyze the accuracy of the residues have provided much useful information for determining the most probable fault of the generator.

\section{MEASURES OF ACCURACY OF THE RESIDUES}

As said above, the error between the theoretical vibrations obtained with the model and the experimental vibrations is called residue. A relative estimate of the residue, $\varepsilon_{1}$, can be obtained using the following expression:

$$
\varepsilon_{1}=\sqrt{\frac{\left(X_{t h}-X_{e x}\right)^{* T}\left(X_{t h}-X_{e x}\right)}{X_{e x}^{* T} \cdot X_{e x}}}
$$

where $*$ indicates complex conjugation and $X_{e x}$ is the vector of the experimental vibrations, while $X_{t h}$ is the corresponding vector of the theoretical vibrations obtained with the model. The machine fault is identified by minimizing the residue; therefore, the most probable fault should be that one with which the lowest residue is associated. As the identification procedure can be time consuming, only the experimental data collected for a limited number of suitable rotating speeds of the machine are analyzed. Sometimes, this strategy is also necessary to avoid using an overabundance of repeated observations in the identification procedure as this could cause numerical problems or identification errors. Obviously, different selections of the rotating speeds, that is different subsets of experimental data, can give quite different results of the fault identification. For instance, if the model does not simulate the response of the system very well near the critical speeds, the selection of vibration data measured in speed ranges near the resonances should be avoided. The ex- citations obtained with the identification procedure can be used to evaluate the system response at any rotating speed associated with the experimental data. This allows a further residue estimation, $\varepsilon_{2}$, to be evaluated. Depending on the choice of the data used for the fault identification, sometimes, although a very low value of the residue $\varepsilon_{1}$ is obtained, the residue estimation $\varepsilon_{2}$ is significantly higher. This is a symptom of poor accuracy of the fault identification. This can be the consequence of a wrong choice in the type of fault as well as in a poor adequacy of the model.

Further indices allow the above-mentioned causes of very different values of the residues $\varepsilon_{1}$ and $\varepsilon_{2}$ to be discriminated. As the object of a fault diagnosis is to identify the actual machine malfunction as well as to simulate the system response, it is important to obtain a low value for both the residues $\varepsilon_{1}$ and $\varepsilon_{2}$. Usually, these residues are computed considering the errors evaluated at different degrees of freedom and at different machine rotating speeds. Therefore, they can be called global residues. On the contrary, further residue estimates can be obtained by selecting the vibration data evaluated at each single rotating speed or at each single degree of freedom (d.o.f.). These residue estimates can be denoted with $\varepsilon_{\omega}$ and $\varepsilon_{\text {dof }}$, respectively. They can be very useful to show the dependence of the error on the rotating speed and the measurement point. In the case of an ideal identification of the fault, the residues should be scarcely affected by the speed value and the d.o.f.. Owing to this, the analysis of the curves of the residue $\varepsilon_{\omega}$ vs. the rotating speed, as well as the curve of $\varepsilon_{\text {dof }}$ vs. the d.o.f., can give very important information on the accuracy of the identified fault and the model adequacy. In general, the ratios between the standard deviations of the residues $\varepsilon_{\omega}$ and $\varepsilon_{\text {dof }}$ and their respective mean values represent good measures of the identification accuracy. The residues are a normalized evaluation of the error between theoretical and experimental vibrations. With reference to this, it is important to consider that the amplitude of the machine vibrations can be quite different depending on the rotating speed and the measurement point. Therefore, the analysis of the curve of the absolute error vs. the rotating speed can point out some information that is masked by the curves of the normalized residues.

In the end, interesting results can be obtained by a correlation analysis between theoretical and experimental data. The real and imaginary parts of all the theoretical and the experimental vibrations can be stored into single locations of the two real vectors $X_{t h}$ and $X_{e x}$, respectively. Then, the Modal Scale Factor (MSF) and the index called Modal Assurance Criterion (MAC) can be evaluated as follows:

$$
\begin{gathered}
\text { MSF }=X_{t h}^{T} X_{e x} / X_{t h}^{T} X_{e x} \\
\text { MAC }=\frac{\left|X_{e x}^{T} X_{t h}\right|^{2}}{\left(X_{e x}^{T} X_{e x}\right)\left(X_{t h}^{T} X_{t h}\right)}
\end{gathered}
$$

In general, the two indices MSF and MAC are used in modal analysis and model updating; however, they also can be applied 
to many other problems. If $X_{t h}$ coincides with $X_{e x}$ the value of the indexes MSF and MAC is 1 . As the identification procedure is based on a least squares method, in this application the MSF value is always very near to 1 . On the contrary, the MAC index gives a measure of the dispersion of the data from the straight line whose angular coefficient is the MSF value. In addition to this, a linear regression analysis of the data contained in the vectors $X_{t h}$ and $X_{e x}$ can be carried out. The difference between the angular coefficient of the regression line and the ideal unity value is a further measure of accuracy of the fault identification. In the end, an additional information is represented by the value of the standard deviation, $\sigma_{r}$, of the errors obtained with the linear regression analysis.

\section{CASE STUDY}

In order to carry out a further validation of the methods developed for evaluating the accuracy of fault identifications, the experimental vibrations of the generator of a co-generation unit have been analyzed. The coupling between generator and gas turbine was provided by a gearbox whose wheels were mounted on a quill-shaft. Figure 1 shows the finite element model of the generator. The rotor was supported on two fluid-film journal bearings (\#1, \#2) that were equipped with two radial XY proximity probes. Bearing \#2 was mounted at the driven end of the generator, beyond the flange of the rigid coupling, that is on a gear shaft.

Owing to the flexibility of the quill-shaft, the influence of the gear shafts on the dynamic behavior of the generator has been neglected, therefore, only the generator and a portion of a gear shaft have been modeled. The machine vibrations were continuously analyzed by a condition monitoring system. Figure 2 shows the Bode plot of the synchronous vibrations (1X) measured on bearing \#1, in vertical direction, during a rundown (Case A) and two runups (Cases B and C). The curves of both amplitude and phase show significant differences in the machine behavior that occurred during the three transients, especially in the speed range near the first balance resonance of the rotor (1740 rpm). Before the first runup (Case B) the machine was rolled very slowly on the turning gear for a long time. On the contrary, the second runup (Case C) was carried out at the end of a two day's downtime without a suitable rotor straightening on the turning gear. During this runup, on bearing \#1, the $1 \mathrm{X}$ vertical vibrations reached $206 \mu \mathrm{m}$ pk-pk at $1740 \mathrm{rpm}$. Also on bearing $\# 2$, the $1 \mathrm{X}$ vibrations measured during the same speed

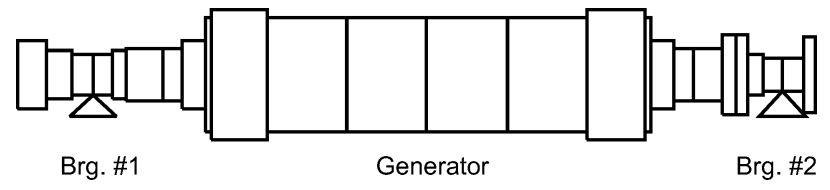

FIGURE 1

Finite element model of the generator rotor.
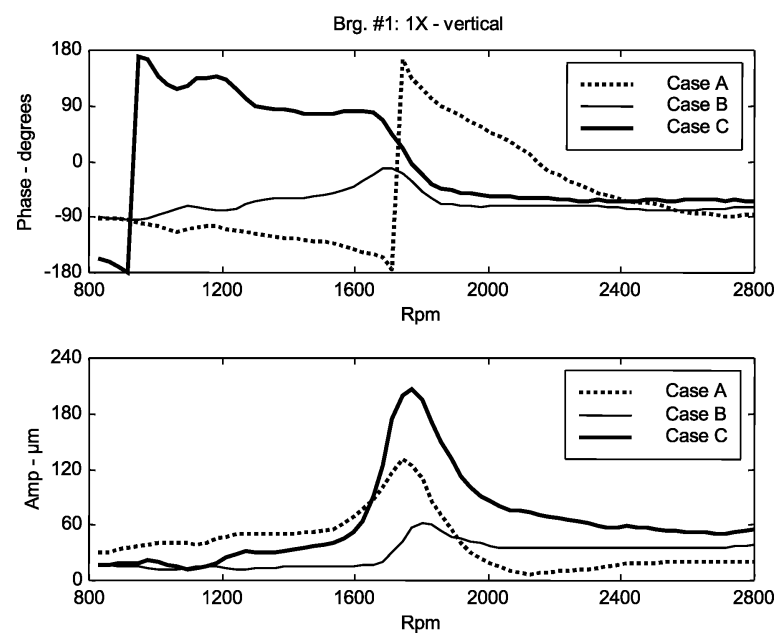

FIGURE 2

Bode plot of 1X transient vibrations measured on bearing \#1, in vertical direction, during different speed transients (Case A: rundown; Cases B and C: runups).

transients (A, B, and C), showed very different amplitudes when approaching the first critical speed. This abnormal behavior was the symptom of a machine malfunction. In order to assure the machine health monitoring it was necessary to identify the actual type of the fault as well as to evaluate its severity and position.

The vibrations caused only by the fault have been evaluated by subtracting the $1 \mathrm{X}$ vectors measured during the runup $\mathrm{B}$ from those measured during the transients $\mathrm{A}$ and $\mathrm{C}$, respectively. In this article, these vibrations will be called additional vibrations as it has been assumed that they are mainly due to the machine fault.

The vibration data collected during various runups and rundowns were analyzed. This investigation showed that the dynamic behavior of the generator was sufficiently repetitive when considering the vibrations measured during speed transients occurring in similar thermal states of the machine. In general, when approaching the first balance resonance, the $1 \mathrm{X}$ vibrations measured during the rundowns occurred in a steady thermal state and with a warm machine were significantly higher than those measured during most of the runups that, usually, are carried out with a cold machine. However, during some runups occurred after a short operating period on the turning gear, the $1 \mathrm{X}$ vibrations showed very high levels when passing through the first critical speed of the generator rotor. Also in these cases, when the machine reached the operating speed $(3000 \mathrm{rpm})$, the vibration amplitudes were fairly low (30-60 $\mu \mathrm{m})$.

Figure 3 shows the Bode plot of the additional vertical vibrations evaluated on bearing \#1 for two couples of speed transients (A-B, C-B). Although the vibrations measured during the two transients $\mathrm{A}$ and $\mathrm{C}$ are quite different both in amplitude and phase, the two amplitude curves of the additional vibrations are very similar and show a quite evident peak near the first critical 

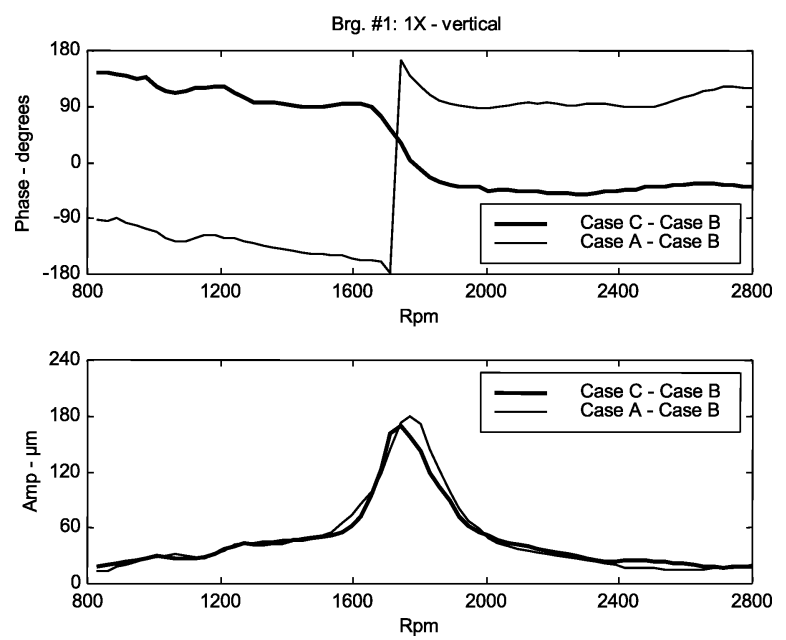

FIGURE 3

Bode plot of $1 \mathrm{X}$ additional vibrations evaluated on bearing \#1 in vertical direction.

speed. Moreover, in the range above $2000 \mathrm{rpm}$, the vibration amplitude does not increase with the rotating speed. On the contrary, the phase curves are shifted by nearly $180^{\circ}$ over the complete speed range from 800 to $2800 \mathrm{rpm}$.

The additional vibrations illustrated in Figure 3 show that the sensitivity of the generator to the malfunction that occurred in the operating condition, and that affects the transient response during the rundowns, was very similar to the sensitivity of the generator to the rotor bow induced by the weight when the machine was stopped. This information provided by the analysis of the additional vibrations is very important for the identification of the type of the machine malfunction. In addition to this, in the operating condition the $1 \mathrm{X}$ vibrations of the generator showed to be a little influenced by the value of the exciter current. However, the assumption of the presence of a short-circuit in the rotor windings was discarded because the generator had been subjected to a recent scheduled maintenance during which electrical tests had been carried out. On the basis of the symptoms described above, it has been possible to suppose that the rotor was subjected to a bow induced by unsymmetrical axial forces caused, for instance, by an excessive or an unsymmetrical thermal expansion of the copper bars mounted inside the generator slots. Conversely, when the operating time on turning gear is too short to cause a complete straightening of the generator rotor, high vibrations occur during the runups, especially when approaching the first balance resonance. In the following, the identification of the machine malfunction has been carried out considering only the additional vibrations evaluated on the basis of the transients $\mathrm{B}$ and $\mathrm{A}$, that is a rundown and a subsequent runup carried out with a straightened rotor.

A rotor bow can be simulated by applying two opposite bending moments, having the same amplitude, to suitable nodes of the FE model of the rotor. The object of the diagnostic technique that has been used was to identify the amplitude and position

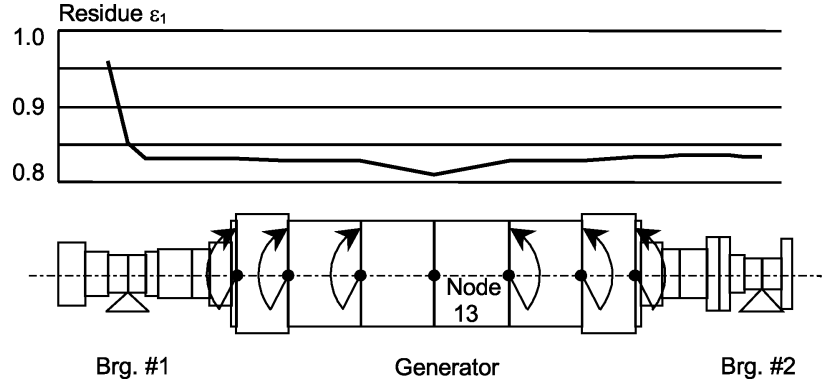

FIGURE 4

Dependence of the global residue $\varepsilon_{1}$ on the axial position of the two bending moments.

of the bending moments that minimize the error between the experimental vibrations and those evaluated with the model. The middle part of the generator rotor, where the copper bars are mounted, has been modeled with an even number of finite elements, equal to six, so that a node (no. 13) was located in the mid-span of the rotor windings (Figures 1 and 4). These six elements were symmetrical with respect to the central node (no. 13). If the fault was really a rotor bow induced by thermal effects in the windings, it was rather probable that the two equivalent bending moments should have been applied to the ends of the windings, or near the retaining rings, in rotor sections symmetrical in respect to the mid-span of the windings.

When the fault is modeled with two opposite moments the identification of the minimum residue is obtained by considering groups of different numbers of adjacent finite elements whose extreme nodes are the points to which the bending moments are applied. Obviously, the mean position of each group of FE is also changed along the rotor. The minimum residue obtained with a complete analysis gives the best estimate of the moments. Then, this residue can be compared with those evaluated by considering other types of faults in order to evaluate the most probable fault.

Vibration data associated with 10 rotating speeds equally spaced in the range from 800 to $2800 \mathrm{rpm}$ have been used to identify the two bending moments. This investigation has been carried out by analyzing the generator vibrations measured both in vertical and horizontal direction. The minimum residue $\varepsilon_{1}$ has been obtained by considering a group of four finite elements whose mean position is just in the mid-span of the rotor windings while the nodes to which the bending moments are applied are not exactly at the extremes of the windings but, anyhow, in their vicinity (nodes 11 and 15). Figure 4 shows the dependence of the residue $\varepsilon_{1}$ on the position of two opposite bending moments applied at the extreme nodes of different groups of four adjacent finite elements.

As discussed above, during the identification procedure, bending moments also have been applied to groups of different numbers of adjacent finite elements. In this analysis, for each number of elements the minimum residue corresponded to a group of FE centerd around the node no. 13, that is a node located in the mid-span of the rotor windings. The values of the 
TABLE 1

Measures of accuracy of the fault identification

\begin{tabular}{lccc}
\hline & \multicolumn{3}{c}{ Number of finite elements } \\
\cline { 2 - 4 } & 6 & 4 & 2 \\
\hline Extreme nodes & $10 / 16$ & $11 / 15$ & $12 / 14$ \\
Residue $\varepsilon_{1}$ & 0.8346 & 0.8107 & 0.8135 \\
Residue $\varepsilon_{2}$ & 0.8440 & 0.8273 & 0.8290 \\
MAC & 0.6635 & 0.6992 & 0.6956 \\
\hline
\end{tabular}

minimum residues associated with groups of 2, 4, and 6 elements are shown in Table 1. Although the differences between these residues are very little, the minimum residue is one that is associated with a group of four elements.

Figures 5 and 6 show the Bode plot of the $1 \mathrm{X}$ vibrations evaluated with the model on bearings \#1 and \#2 using the identified bending moments. In the same figures the numerical response of the system has been compared with the respective experimental one. With regard to the vertical vibrations, the numerical response on both bearings is in good accordance with the experimental behavior. Conversely, the error between the numerical response in the horizontal direction and the respective experimental vibrations is not negligible, especially in the range around the balance resonance. Concerning this, it is important to consider that on both the bearings, when passing through the first balance resonance, the $1 \mathrm{X}$ vertical vibrations were up to three times higher than the horizontal vibrations. In addition to this, the horizontal additional vibrations do not show a significant and evident positive peak in the speed range near the first balance resonance of the generator. This abnormal behavior can be induced by a machine misalignment as well as by the bearing characteristics. In order to simulate this behavior with care,
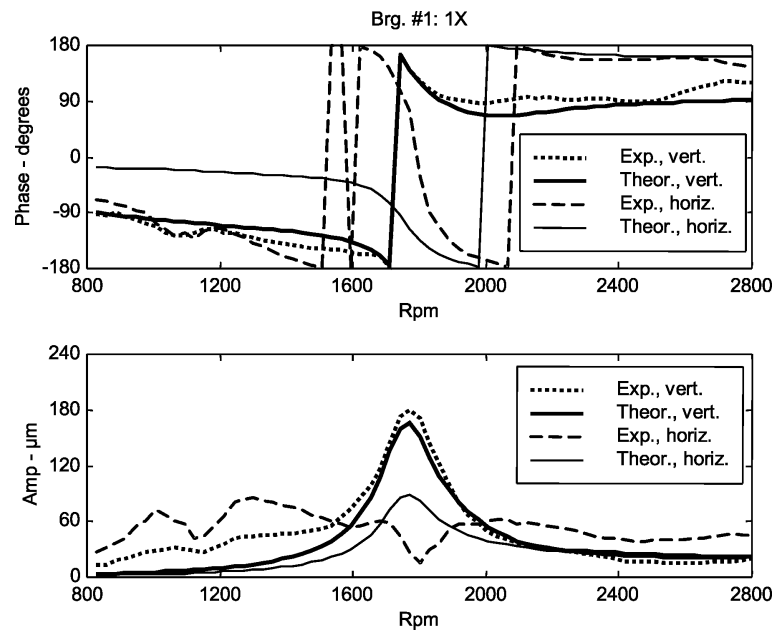

FIGURE 5

Comparison between the experimental $1 \mathrm{X}$ vibrations measured on bearing \#1 and the respective numerical response obtained with the identified bending moments.
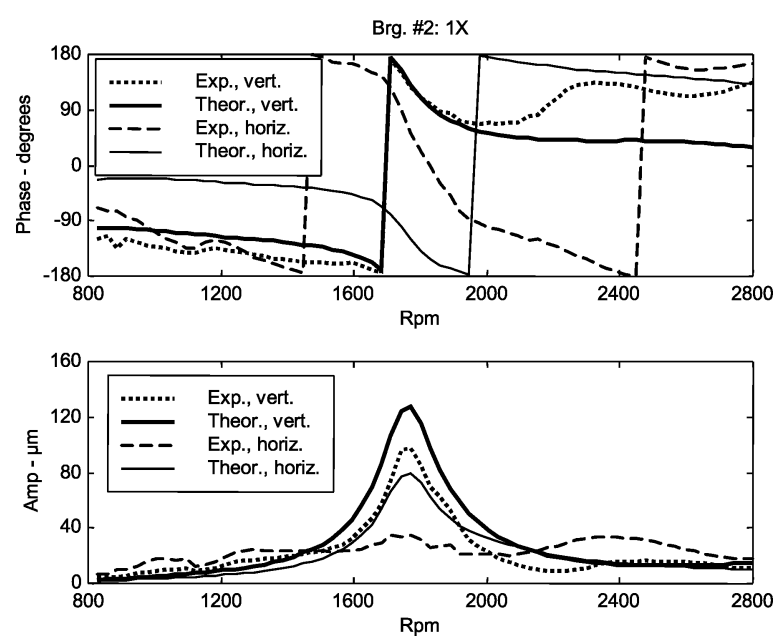

FIGURE 6

Comparison between the experimental $1 \mathrm{X}$ vibrations measured on bearing \#2 and the respective numerical response obtained with the identified bending moments.

a preliminary tuning of the model has been carried out; however, a further optimization of some bearing coefficient would be required. The identified bending moments allow the highest vibrations to be simulated with good accuracy over the complete speed range from 800 to $2800 \mathrm{rpm}$.

A further residue estimate, $\varepsilon_{2}$, has been carried out by considering the identified moments and all the available vibrations data associated with 68 rotating speed values almost uniformly spaced in the range of 800 to $2800 \mathrm{rpm}$. Some estimates of the residue $\varepsilon_{2}$ are shown in Table 1 . The values of the residues $\varepsilon_{1}$ and $\varepsilon_{2}$ are very similar although they have been obtained using quite a different number of vibration data. This result shows that the fault identification was not significantly influenced by a "biased" choice of the vibration data. The residues $\varepsilon_{1}$ and $\varepsilon_{2}$ are not very low, however, the analysis of the residues $\varepsilon_{\text {dof }}$, calculated considering the vibrations evaluated at each measurement point, shows that the residues associated with the vertical vibrations are rather low and are significantly lower than the residues associated with the horizontal vibrations (Figure 7).

The four residues $\varepsilon_{\text {dof }}$ summarize the results obtained by comparison between the experimental and numerical responses shown in Figures 5 and 6 . Another residue estimate has been carried out by considering, separately, the vibration data associated with each of the 68 rotating speed values. Figure 8 shows the dependence of the residues $\varepsilon_{\omega}$ on the rotating speed. At first, both vertical and horizontal vibrations on the two bearings have been considered, that is four degrees of freedom. The bold line in Figure 8 (Case: 4 dof) shows that in the speed range below the first balance resonance the residues are rather high while in the range above $1700 \mathrm{rpm}$ they decrease significantly up to a mean value of about 0.6 . This behavior primarily can be ascribed to errors in the evaluation of some bearing coefficient, mostly in the horizontal direction. 


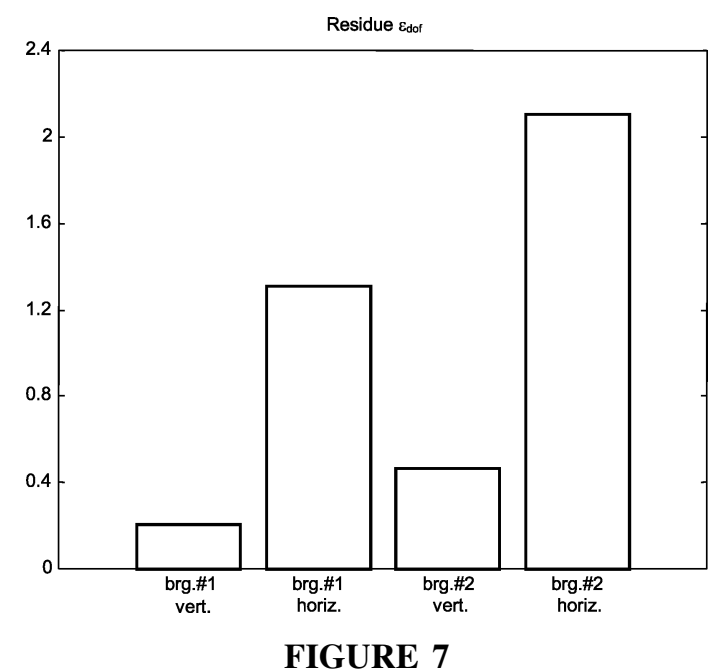

Dependence of the residues, $\varepsilon_{\mathrm{dof}}$, on the degree of freedom.

A further estimate of the residue $\varepsilon_{\omega}$ has been carried out by considering only the vertical vibrations on the two bearings, that is, by taking into account only two degrees of freedom. This choice was due to the fact that, in this case, vertical vibrations were more significant. The dependence of this residue on the rotating speed is shown by the thin line in Figure 8 (Case: 2 dof). Contrary to many usual results, the curve of this residue shows a very evident negative peak just in the speed range around the first critical speed of the generator. The low values of the residues inside this speed range are a clear indication of the accuracy of the fault identification.

The residues obtained with this analysis increase in the ranges below and above the critical speed only because of the low amplitudes of the additional vibrations that occurred outside the resonance peak. In fact, as shown by Equation (1), the residue is a relative estimate of the error. The dependence of the absolute error on the rotating speed is shown in Figure 9. If only the verti-

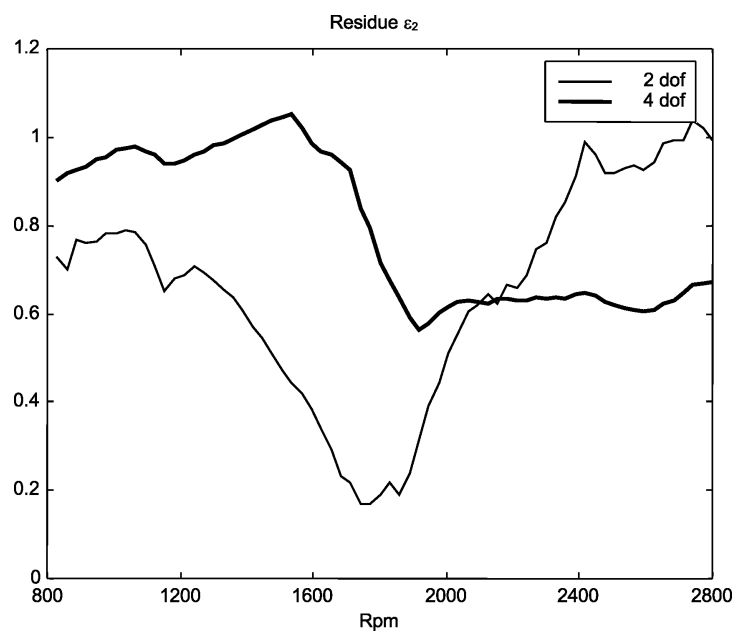

FIGURE 8

Dependence of the residues $\varepsilon_{\omega}$ on rotating speed.

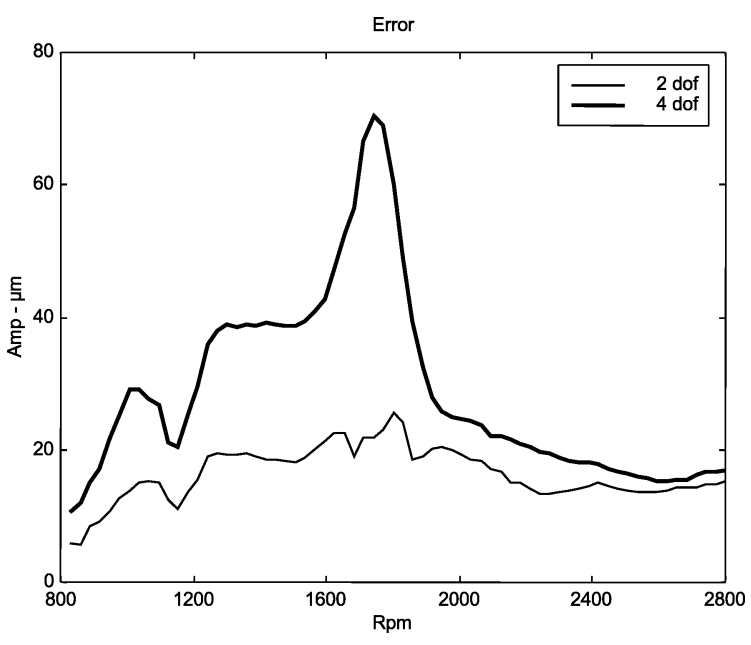

FIGURE 9

Absolute error vs. rotating speed.

cal vibrations are considered (Case: 2 dof, thin line), the absolute error is nearly constant over the complete speed range from 800 to $2800 \mathrm{rpm}$. This causes the relative error $\varepsilon_{\omega}$ to increase when the vibration amplitudes decrease significantly.

Moreover, it is necessary to consider that the additional vibrations should be caused only by the fault that must be identified. Therefore, the two speed transients used to evaluate the additional vibrations must be chosen with care; however, when the amplitude of the additional vibrations is rather low, the error in the phase evaluation can be important because these low vibrations can be due also to other secondary unexpected causes.

The procedure developed by the authors for analyzing the residues obtained with the fault identification method allows the most important characteristics of the residues to be emphasized, so that interesting information about the reliability of the identified fault can be obtained.

Figure 10 shows the results of a regression analysis carried out between experimental and numerical vibration data. In accordance with the results described above, the larger dispersion of the data is associated with the vibrations that show the lowest levels. The estimates of the MAC index as well as some results of a statistical analysis of the residues $\varepsilon_{\omega}$, obtained with the identified moments, are reported in Table 2.

In this case study the fault has occurred on a generator and not on a turbine; this fact as well as some significant symptoms suggested that the fault was a rotor bow. In order to evaluate the

TABLE 2

Measures of accuracy of the fault identification

\begin{tabular}{lcccc}
\hline $\begin{array}{l}\text { Degrees } \\
\text { of freedom }\end{array}$ & Residue $\varepsilon_{1}$ & $\begin{array}{c}\text { Residue } \varepsilon_{\omega} \\
\text { mean value }\end{array}$ & $\begin{array}{c}\text { Residue } \varepsilon_{\omega} \\
\text { standard dev. } \sigma_{\omega}\end{array}$ & MAC \\
\hline 4 d.o.f. & 0.8273 & 0.7917 & 0.1725 & 0.6992 \\
2 d.o.f. & 0.3647 & 0.6508 & 0.2457 & 0.9401 \\
\hline
\end{tabular}




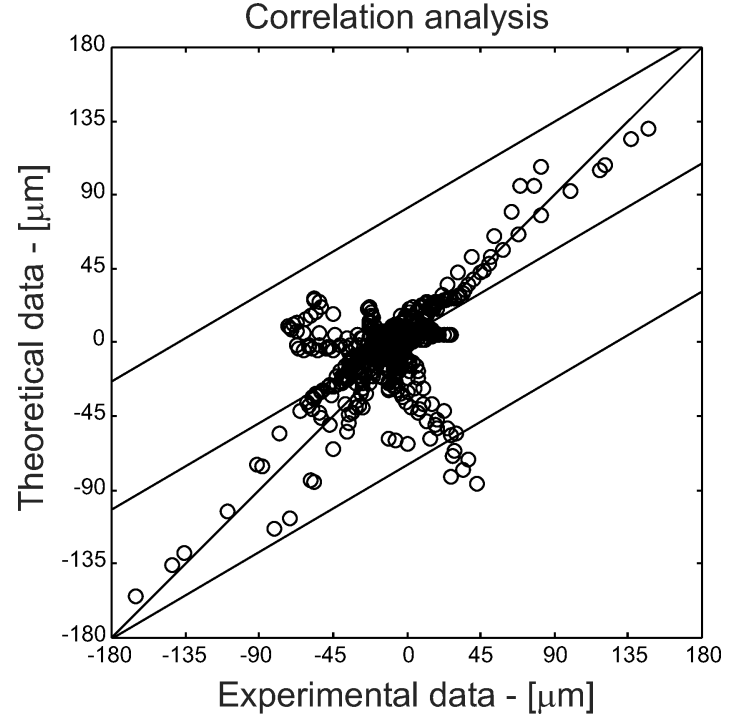

FIGURE 10

Regression analysis.

capabilities of the techniques developed to analyze the residues associated with the identified faults, a further investigation has been carried out considering a local unbalance as the cause of the additional vibrations of the generator.

The identification of position and magnitude of the equivalent force due to a rotor unbalance has been obtained with the same subset of vibration data previously used to identify the bending moments. These data were associated with 10 rotating speeds equally spaced in the range from 800 to $2800 \mathrm{rpm}$. Figure 11 shows the dependence of the residue $\varepsilon_{1}$ on the axial position of the unbalance.

The minimum residue obtained with this analysis has been evaluated by applying a local unbalance to the node no. 13, that is the node located in the mid-span of the rotor windings. Afterwards, the identified unbalance has been used to evaluate the residue $\varepsilon_{2}$, obtained considering all the available vibration data. The values of the two residues $\varepsilon_{1}$ and $\varepsilon_{2}$ were very similar $\left(\varepsilon_{1}=1.1463 ; \varepsilon_{2}=1.0779\right)$; moreover, they are significantly higher than the respective residues obtained with the identification of the rotor bow (Table 1).

Figures 12 and 13 show the Bode plot of the $1 \mathrm{X}$ vibrations evaluated with the model on bearings \#1 and \#2 using the iden-

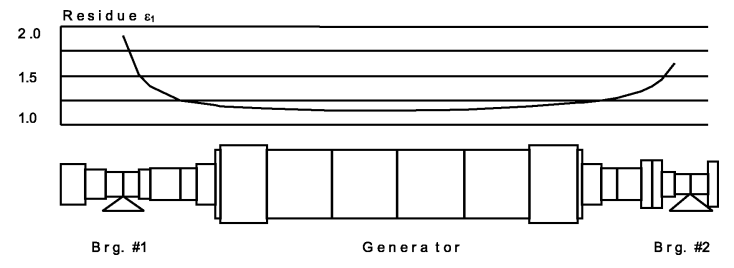

FIGURE 11

Dependence of the global residue $\varepsilon_{1}$ on the position of the local unbalance.
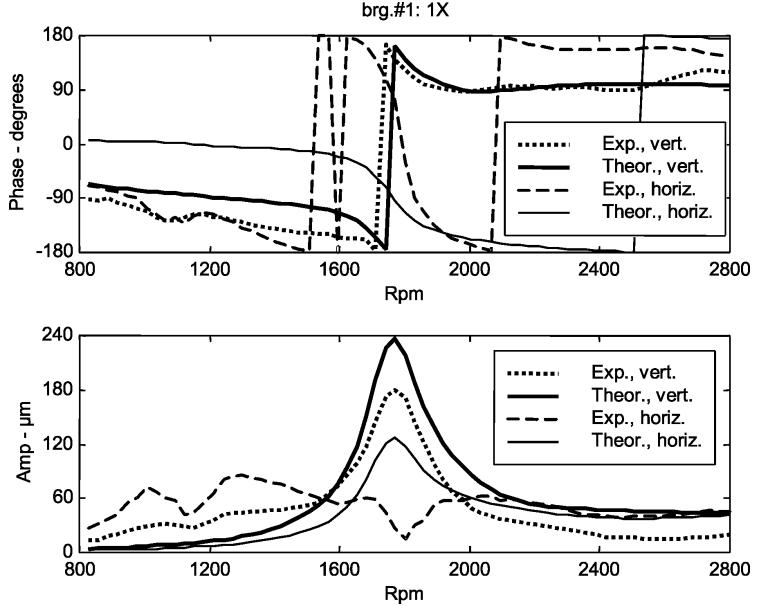

FIGURE 12

Comparison between the experimental $1 \mathrm{X}$ vibrations measured on bearing \#1 and the respective numerical response obtained with the identified unbalance.

tified unbalance. In the same figures the numerical response of the system has been compared with the respective experimental one. Figures 12 and 13 show that even the best identified local unbalance causes significant errors over the complete speed range from 800 to $2800 \mathrm{rpm}$, not only in the range outside the region of the resonance peak.

Figure 14 shows the curves both of the relative and absolute errors vs. the generator rotating speed. These curves, which have been obtained considering both vertical and horizontal vibrations, can be compared with the respective ones shown in Figures 8 and 9 (Case: 4 dof). In general, the absolute and relative errors associated with the identified unbalance are higher than the errors associated with the rotor bow.
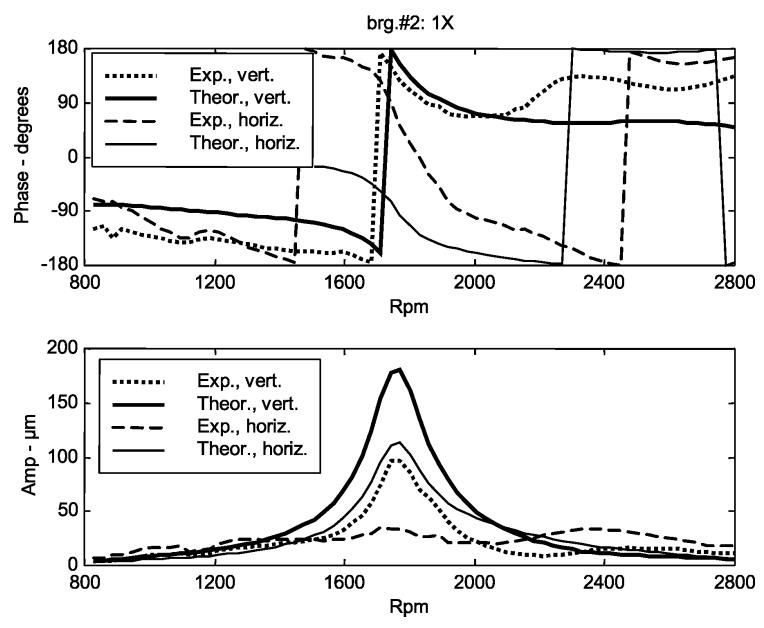

FIGURE 13

Comparison between the experimental $1 \mathrm{X}$ vibrations measured on bearing \#2 and the respective numerical response obtained with the identified unbalance. 

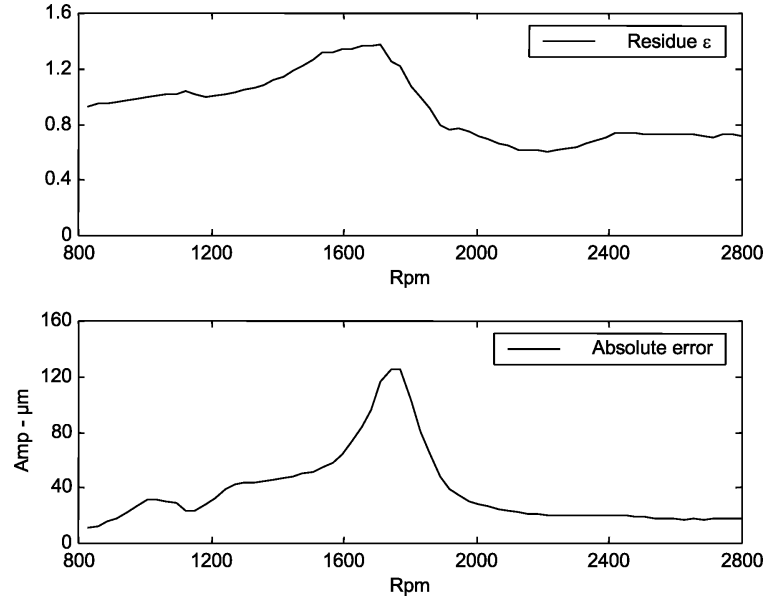

FIGURE 14

Relative and absolute errors vs. the generator rotating speed.

By considering the characteristics of the machine and fault symptoms, the type of the identified fault and the position of the equivalent bending moments along the rotor are probable. Although the results of the fault identification are rather good, a further refining of the tuning of the bearing coefficients could allow a more accurate identification of the moments to be obtained.

\section{CONCLUSIONS}

In the past, some methods have been developed by the authors for analyzing the accuracy of the results obtained with modelbased techniques aimed to identify faults in rotating machinery. The capabilities of these methods had been already shown with previous studies carried out using only machine responses simulated with mathematical models. This article has shown that the developed methods also can be successfully used for the analysis of experimental vibrations of real machines.

These proposed diagnostic techniques have shown to be able to give useful information for identifying the most probable fault also when different sets of equivalent excitations which can induce fault symptoms very similar to the real one are considered. Therefore, these methods are helpful to discriminate the actual fault from other wrong but probable faults. In addition to this, these methods have shown to be very useful in detecting lacks of accuracy in the model of the fully assembled machine as well as errors in the experimental vibration data.

\section{NOMENCLATURE}

$\varepsilon_{1} \quad$ residue evaluated with the vibration data associated with a restricted number of rotating speeds

$\varepsilon_{2}$ residue evaluated with the vibration data associated with all the available rotating speeds measured during transients

$\varepsilon_{\text {dof }}$ residue evaluated considering the vibration data associated with a single degree of freedom

$\varepsilon_{\omega} \quad$ residue evaluated considering the vibration data associated with a single rotating speed

$X_{e x}$ experimental vibration vector

$X_{t h}$ theoretical vibration vector

\section{REFERENCES}

Bachschmid, N., and Pennacchi, P. 2000. Model based malfunction identification from bearing measurements, IMechE-7th International Conference on Vibrations in Rotating Machinery, September 12-14, University of Nottingham, UK, 571-580.

Bachschmid, N., Pennacchi, P., Tanzi, E., and Vania, A. 2000. Accuracy of modelling and identification of malfunctions in rotor systems: experimental results. Journal of the Brazilian Society of Mechanical Sciences, XXII, 3, 423-442.

Bachschmid, N., Vania, A., Tanzi, E., and Pennacchi, P. 1999. Identification and simulation of faults in rotor systems: experimental results, EURO DINAME 99, Günzburg, Germany, 3-11.

Markert, R., Platz, R., and Seidler, M. 2000. Model based fault identification in rotor systems by least squares fitting, ISROMAC-8 Conference, March 26-30, Honolulu, Hawaii, 901-907.

Pennacchi, P., and Vania, A. 2001a. Measures of accuracy of model based identifications of faults in rotormachinery, 15th AIMETA Congress of Theoretical and Applied Mechanics, Taormina, Italy, SP_ME_48.

Pennacchi, P., and Vania, A. 2001b. Measures of accuracy of model based diagnosis of faults in rotormachinery, COMADEM 2001, Manchester, UK, 873-880.

Platz, R., Markert, R., and Seidler, M. 2000. Validation of online diagnostics of malfunctions in rotor systems, IMechE-7th International Conference on Vibrations in Rotating Machinery, September 12-14, University of Nottingham, UK, 581-590. 

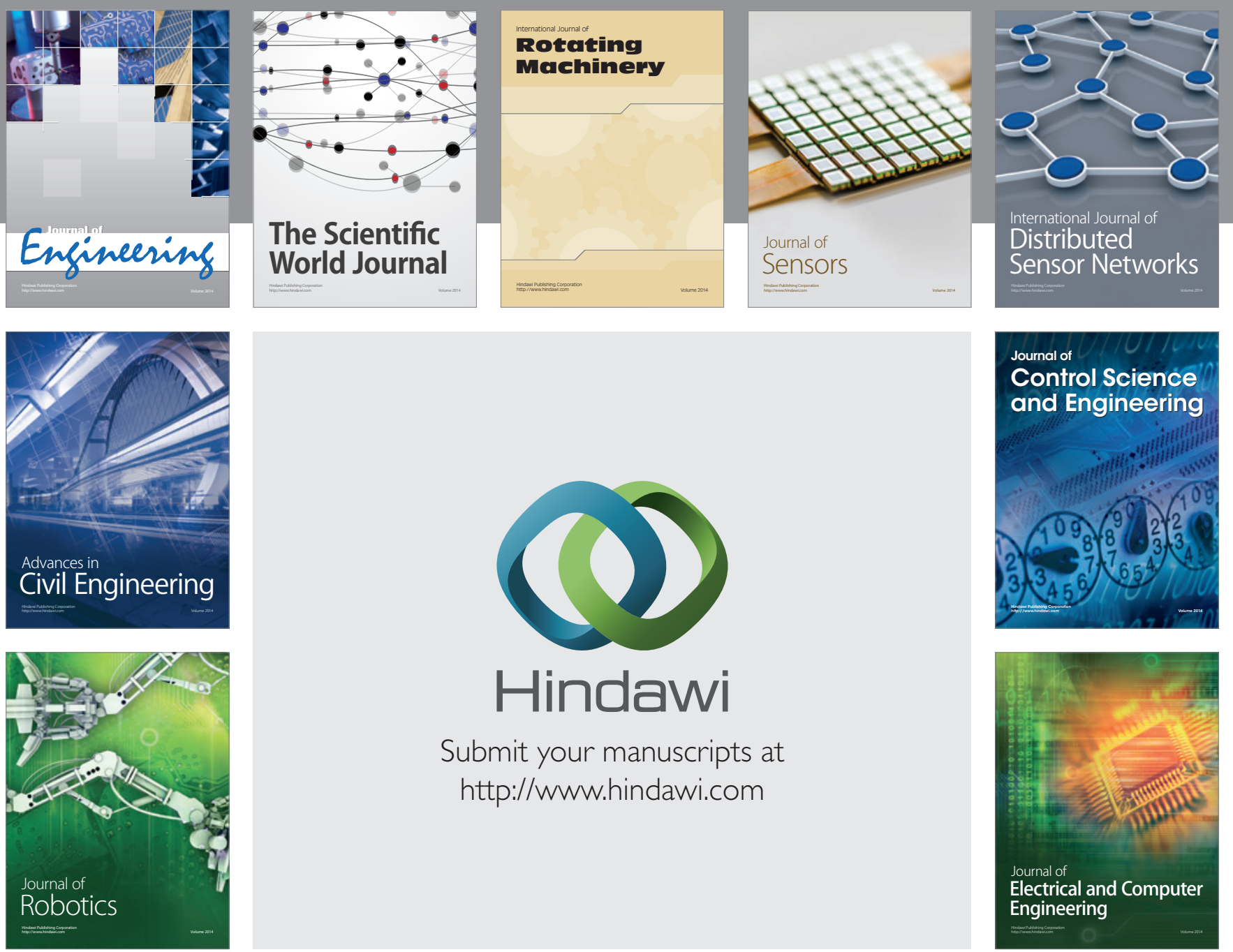

Submit your manuscripts at

http://www.hindawi.com
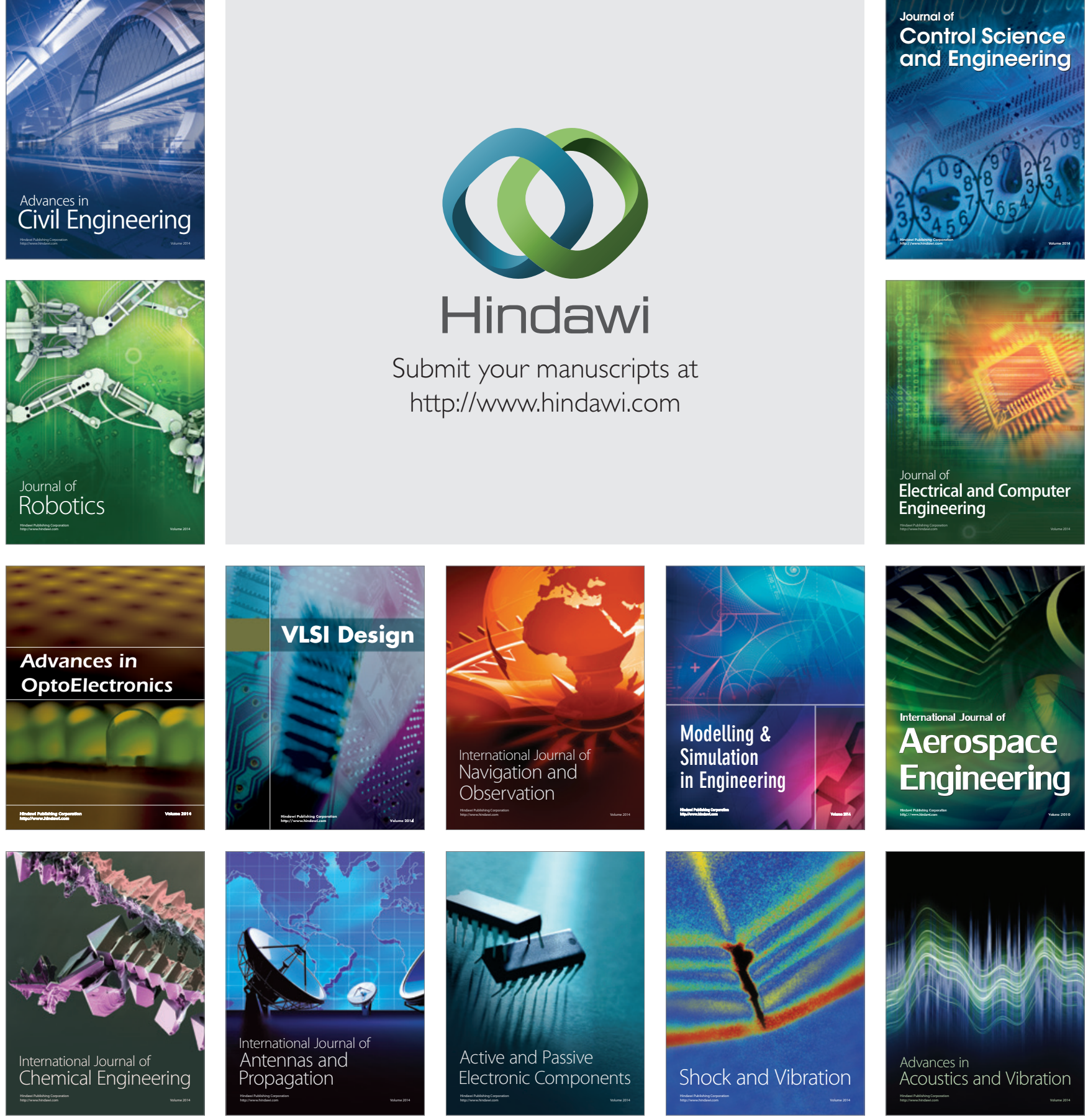